\title{
Prognostic factors for severe pulmonary involvement in systemic sclerosis
}

\author{
Alexandra-Daniela Radu ${ }^{1,2}$, Ana Maria Gheorghiu ${ }^{1,2}$, Raida Oneata ${ }^{1,2}$, Alina Soare ${ }^{1,2}$, \\ Ruxandra Dobrota ${ }^{1,2}$, Stefania Magda ${ }^{2,3}$, Tudor Constantinescu ${ }^{2,4}$, Ruxandra Jurcut ${ }^{2,5}$, \\ Roxana Sfrent-Cornateanu ${ }^{2,6}$, Mihai Bojinca ${ }^{1,2}$, Victor Stoica ${ }^{1,2}$, Carina Mihai ${ }^{1,2}$ \\ ${ }^{1}$ Internal Medicine and Rheumatology, Cantacuzino Hospital, Bucharest, Romania \\ 2"Carol Davila" University of Medicine and Pharmacy, Bucharest, Romania \\ ${ }^{3}$ Emergency University Hospital, Bucharest, Romania \\ 4"Marius Nasta" Institute for Cardiovascular Diseases, Bucharest, Romania \\ 5"Prof. Dr. C.C. Iliescu" Institute for Cardiovascular Diseases, Bucharest, Romania \\ 6Physiopathology and Immunology Department, Bucharest, Romania
}

\begin{abstract}
Background. Lung involvement is the main disease related death cause in patients with systemic sclerosis (SSc). The most frequent lung manifestations in SSc are interstitial lung disease (ILD) and pulmonary arterial hypertension (PAH).

Objectives. Evaluation of lung involvement in patients with SSc and identification of predictive factors for severe lung involvement.

Patients and methods. All patients with SSc of the EUSTAR100 center, having at least two visits between 2004 and 2016, were included. Survival status, cause of death, dyspnea, ILD on thorax radiography or high resonance thorax computer tomography (HRCT) and lung function tests were recorded during the entire follow-up. Severe lung involvement was defined as severe or end-stage lung involvement on the Medsger severity scale at any time during follow-up, or death. Cox proportional hazards regression was used in univariate and multivariate analysis to identify prognostic factors.

Results. 89 patients were included $(12.4 \%$ males, mean age \pm SD $49.2 \pm 12.2$ years, disease duration $4.1 \pm 7.5$ years), with a follow-up duration up to 13 years. 14 deaths were reported, half due to lung involvement (4 deaths due to ILD, 3 deaths due to PAH). Pulmonary involvement was identified in a large proportion: at first visit 28/55 present ILD on thorax radiography, 7/12 on HRCT scans; at the most recent visit 41/71 present ILD on Xray, $18 / 24$ on HRCT scans. At least $10 \%$ decrease of pulmonary diffusion capacity for carbon monoxide (DLCO) and of forced vital capacity (FVC) was observed in 18/32 and 8/35 respectively; 24/48 developed severe or endstage pulmonary involvement on the Medsger scale or have died. Risk factors for severe lung involvement were age $>60$ years, disease duration $<3$ years and diffuse cutaneous subset.

Conclusion. SSc often presents unfavourable disease course, mostly due to pulmonary involvement. While half of the deaths reported were due to lung involvement, only about half of the patients presenting ILD-typical findings on Rx develop end-stage lung involvement or death. It is of great importance to screen at baseline for ILD and $\mathrm{PAH}$, following up annually, even while patients are asymptomatic.
\end{abstract}

Keywords: systemic sclerosis, prognostic factors, lung involvement

\section{INTRODUCTION AND OBJECTIVES}

Systemic sclerosis (SSc) is a chronic systemic autoimmune disease with variable course. ILD and PAH are the most common types of lung involvement in SSc patients, as well as the main SSc-related mortality and morbidity causes in these patients (1).

SSc-ILD is characterized by inflammation or fibrosis of the lung parenchyma, possibly leading to impaired gas exchange, respiratory failure and eventually death. Its pathogenesis is not completely understood, with an immunological injury to pulmonary capillaries playing a potential role (2).

Dyspnea is the main symptom in SSc-ILD, but as it is neither sensitive nor specific, screening strategies for lung involvement are recommended in all SSc patients. The evaluation of respiratory symptoms, HRCT and pulmonary function tests (PFT) is 
a well accepted method for SSc-ILD diagnosis, while transthoracic echocardiography (TTE) is of high importance in PAH screening (3).

This study aims to asses the proportion of patients presenting lung involvement, identify the main death causes as well as prognostic factors for worsening of lung function, in a Romanian single-center cohort of patients with SSc.

\section{PATIENTS AND METHODS}

All patients diagnosed with SSc by expert opinion, who attended the EUSTAR100 center between January 2004 and March 2016, presenting at least 2 visits in the clinic were included in this study. All patients have signed informed consent for the recording and analysis of medical data. Patients were screened annually by X-ray, PFT and TTE; HRCT was recommended if PFV showed abnormalities and right heart catheterization recommandation if $\mathrm{PAH}$ was suspected.

We defined severe or end-stage lung involvement on the Medsger severity scale or death (4). Candidate risk factors were selected by clinical judgement among baseline parameters and included: male sex, age $>60$ years, disease duration $<3$ years, anti-centromere antibodies, anti-topoisomerase I antibodies, mRSS $>14$, history of digital ulcers, diffuse cutaneous subset, $\mathrm{FVC}<70 \%$, DLCO $<80 \%$ of predicted, and proteiuria. Risk factors were assessed by univariable Cox proportional hazards regression, with a $\mathrm{p}$ value $<0.05$ considered as statistically significant.

\section{RESULTS}

\section{Demographic data}

The study cohort comprised 89 patients $(12.35 \%$ men, mean age $49.2 \pm 12.2$ years, disease duration $4.1 \pm 7.5$ years), with a follow-up duration up to 13 years. The female:male ratio was about $7: 1$, with age at diagnosis for men $43 \pm 17.74$ years, while for women $44.45 \pm 14.55$ years. $44.94 \%$ of patients were classified as diffuse cutaneous subset, with age at diagnosis for diffuse cutaneous subset $41.45 \pm 12.73$ respectively $46.57 \pm 16.19$ years for limited subset.

TABLE 1. Demographic and clinical data of the patients at enrolment $(n=89)$

\begin{tabular}{|l|c|}
\hline Age, years & $49.2 \pm 12.2$ \\
\hline Age at diagnosis, years & $44.27 \pm 14.88$ \\
\hline Disease duration since diagnosis & $4.1 \pm 7.5$ \\
\hline Male sex & $11(12.35 \%)$ \\
\hline Diffuse cutaneous subset & $40(44.94 \%)$ \\
\hline mRSS, mean; q1;q3 & $8.78 \pm 8.647 ; 2 ; 13$ \\
\hline Interstitial lung disease & $35(39.32 \%)$ \\
\hline Heart involvement & $27(30.3 \%)$ \\
\hline Gastrointestinal involvement & $74(83.14 \%)$ \\
\hline Raynaud's phenomenon & $100 \%$ \\
\hline Renal crisis history & $1(1.12 \%)$ \\
\hline Musculo-articular involvement & $35(39.32 \%)$ \\
\hline
\end{tabular}

\section{Disease evolution and prognostic factors}

Lung involvement was identified in a large proportion: at first visit 28/55 presented ILD on thorax radiography (Rx), 7/12 on HRCT scans; at the last

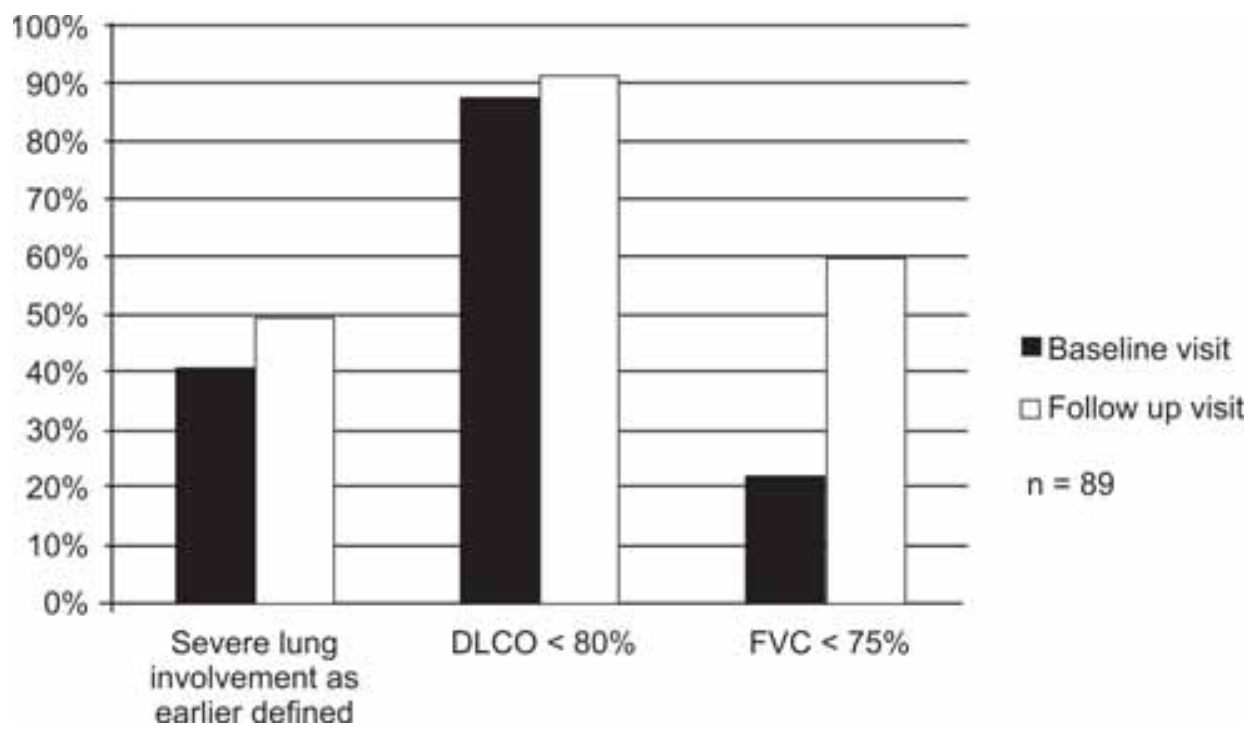

FIGURE 1. Lung involvement at baseline and follow-up visit. Severe lung involvement was defined by the Medsger severity criteria (4) 
visit 41/71 present ILD on X-ray, 18/24 on HRCT scans. At least $10 \%$ decrease of DLCO and FVC was observed in $18 / 32$ and $8 / 35$, respectively: $24 / 48$ developed severe or end-stage pulmonary involvement on the Medsger scale or died. There were 14 reported deaths, in 7 cases death being considered as due to lung involvement (4 deaths due to ILD, 3 deaths due to $\mathrm{PAH})$.

Six of 28 patients who had specific ILD findings on $\mathrm{Rx}$ presented dyspnea at baseline; at the last visit the proportion has risen to almost 50\% (19/41).

Risk factors for severe pulmonary involvement were age $>60$ years, disease duration $<3$ years and diffuse cutaneous subset.

TABLE 2. Risk factors for severe pulmonary involvement (univariable Cox proportional hazards regression). $H R=$ hazards ratio, $\mathrm{Cl}=$ confidence interval

\begin{tabular}{|l|c|c|c|}
\hline \multicolumn{4}{|c|}{ Risk factors for severe pulmonary involvement } \\
\hline & $\mathrm{HR}$ & $95 \% \mathrm{Cl}$ & $\mathrm{p}$ \\
\hline Age $>60$ years & 3.718 & $1.435-9.631$ & 0.007 \\
\hline Disease duration $<3$ years & 45.941 & $5.01-421.284$ & 0.001 \\
\hline Diffuse cutaneous subset & 2.846 & $1.125-7.199$ & 0.027 \\
\hline
\end{tabular}

\section{DISCUSSIONS}

A wide clinical variety is observed in the course of SSc-ILD, with only $21 \%$ of patients with specific ILD findings on Rx presenting dyspnea at baseline, respectively $46 \%$ during follow-up. This shows that patients may be asymptomatic or minimally symptomatic at early stages of mild lung involvement, experiencing symptoms with increase of disease duration, disease worsening or older age. It is thus neccesary to screen at baseline for ILD and PAH, following up annually, even if patients are asymptomatic.

While ILD is a common feature in SSc and the main SSc related death cause (in our study $46.6 \%$ of patients present Rx modifications at last visit), only about half of the patients presenting typicalILD findings on Rx developed severe or end-stage lung in- volvement or died. This might be due to the close screening for lung involvement and starting of immunosupressive treatment with either cyclophosphamide or mycophenolate mofetil as soon as SScILD was diagnosed, respectively with azathioprine as an alternative. There is an underlying necessity of further analysis of potential risk factors at baseline for the worsening of lung function.

Diffuse cutaneous subset, older age and early disease where found to be risk factors for worsening of SSc-lung disease in our study, with similar results found in the reviewed literature. A large study conducted on $900 \mathrm{SSc}$ patients identified male sex, afro-american ethnicity, early disease and primary cardiac involvement due to $\mathrm{SSc}$ as risk factors for severe lung involvement (defined by an $\mathrm{FVC}<50 \%$ of predicted) (5). Different results regarding potential risk factors for SSc-ILD worsening coud be explained by the variability used to define SSc-ILD and number of patients included in the studies.

Limitations of our study were deficiencies in data collecting for the follow up tests, which were carried out in other facilities (with only $37 \%$ and $40 \%$ of patients having both at baseline and at last visit DLCO respectively FVC data), lack of firm classification criteria for SSc-ILD and the limited number of patients included in our study.

\section{CONCLUSIONS}

SSc often presents an unfavourable disease course, mostly due to pulmonary involvement. While half of the deaths reported were due to lung involvement, only about half of the patients presenting typical findings on Rx develop end-stage lung involvement or death. It is of great importance to screen at baseline for ILD and PAH, following up annually, even while patients are asymptomatic. Risk factors for worsening of lung function were age $>60$ years, disease duration $<3$ years and diffuse cutaneous subset.

Conflict of interest: none declared Financial support: none declared

\section{REFERENCES}

1. Bussone G., Mouthon L. Interstitial lung disease in systemic sclerosis, Autoimmune Rev, 2011 Mar;10(5):248-55. doi: 10.1016/j. autrev.2010.09.012. Epub 2010 Sep 21.

2. Kowal-Bielecka O., Furst D.E., Pulmonary Interstitial/vascular involvement. ILD associated with systemic sclerosis, Eular On-line course on Systemis Sclerosis - module no 5a.

3. Schoenfeld S.R., Castelino F.V. Evaluation and management approaches for scleroderma lung disease, Ther Adv Respir Dis.,
2017 Aug;11(8):327-340. doi: 10.1177/1753465817713680. Epub 2017 Jun 16.

4. Medsger T.A. Jr., Bombardieri S., Scorza R., Della Rossa A., Bencivelli W. Assessment of disease severity and prognosis. Clin Exp Rheumatol 2003; 21 (Suppl 29): S42-S46.

5. Steen V.D., Conte C., Owens G.R., Medsger T.A. Jr.: Severe restrictive lung disease in systemic sclerosis. Arthritis and Rheumatism 1994, 37: 1283-1289. 